\title{
Helga Schneider: la ferita aperta della scrittura
}

\section{Francesco Ardolino}

Universitat de Barcelona

\begin{abstract}
In quest'intervista, concessa da Helga Schneider nel novembre 2002, si evidenziano le idee che percorrono tutti i suoi romanzi, dall'impossibilità del perdono fino al rifiuto dell'odio. Schneider mostra gli orrori del nazismo dal di dentro e interpreta il Male a partire dalla banalità delle sue espressioni più quotidiane, illuminando le zone d'ombra celate dal velo della propaganda.
\end{abstract}

Parole chiave: Olocausto, letteratura e impegno, letteratura e pedagogìa sociale.

\begin{abstract}
In this interview, granted by Helga Schneider in November 2002, the ideas that predominante in all her novels are present, from the impossibility of forgiveness to the refusal of hatred. Schneider shows the horrors of Nazism from within and interprets Evil from the viewpoint of its trivial daily expressions, illuminating the shady areas hidden by the veil of propaganda.
\end{abstract}

Key words: Holocaust, literature and political commitment, literature and social pedagogy.

Se non li conoscete guardate il capobanda:

è un boia, un assassino colui che li comanda.

Sull'orbace s'è indossato la camicia e la cravatta

perché resti mascherato tutto il sangue che lo imbratta,

ha comprato un tricolore e ogni volta lo sbandiera

ché si sente un po' l'odore della sua camicia nera,

punta a far l'uomo da bene fino a quando gli conviene [...]

(Fausto AMODEI, Se non li conoscete, 1973)

In occasione della presentazione della traduzione castigliana e catalana di Lasciami andare, madre, ${ }^{1}$ Helga Schneider assicurava, senza titubanze, di non

1. Déjame ir, madre, trad. di Elena de Grau, Barcellona, La Salamandra, 2002; Deixám, mare, trad. di Anna Casassas, Barcellona, Empúries, 2002. 
provare odio nei confronti di una genitrice che, arruolatasi nelle SS, aveva abbandonato marito e figli per diventare guardiana nel campo di sterminio di Birkenau. Di fronte ai giornalisti e a un pubblico esterrefatto, spiegava che l'odio era il principio stesso del Male e che, perciò, andava debellato alle origini. A questa posizione ideologica, peraltro ricollegabile alle dichiarazioni di Primo Levi nell'Appendice a Se questo è un uomo, si aggiunga naturalmente il fattore umano, da cui il disperato tentativo della scrittrice di recidere il cordone ombelicale per ricacciare definitivamente l'orrore.

Il libro è il resoconto implacabile di un dialogo fallito in partenza. Nel 1998, a diciassette anni di distanza da un primo incontro in cui la scoperta della sconvolgente verità le aveva impedito di mantenere un qualsiasi contatto con la madre, Helga Schneider riceve una lettera da una casa di riposo per anziani e accetta di compiere un'ulteriore visita che si trasformerà in un interminabile supplizio. Non c'è solo il documento personale o lo scavo psicologico di un rapporto inesistente: il testo si dispiega di fronte al lettore quasi fosse una partita di scacchi con un finale funesto perché è ancora la madre, malgrado i suoi novant'anni, a condurre il gioco secondo la logica della propria follia.

Un paio d'anni fa, in un'intervista rilasciata alla Repubblica, la scrittrice condensava in poche frasi la mostruosità del personaggio principale: «Condannata a sei anni di carcere dal tribunale di Norimberga, mia madre ne ha scontati solo due, perché ha collaborato con gli inquirenti denunciando i suoi compagni di sterminio. Oltretutto è anche un'opportunista. Sa come la definisce il dossier del Centro Wiesenthal, che da sempre ricerca i criminali nazisti? Bugiarda, fanatica, infida. Ha perfino collaborato agli esperimenti su cavie umane, a Ravensbrück». E viene da pensare, di fronte a queste affermazioni, a tanto revisionismo meschino e comparatistico, che vuole declinare l'Olocausto al plurale per celarne la bestialità e sdoganare vecchie e nuove Destre: basta il brano della canzone di Amodei qui in epigrafe per far accaponare la pelle di fronte alla reiterata insorgenza di certi meccanismi.

Schneider risponde in modo schivo alle domande strettamente letterarie poiché rifiuta l'interferenza dello scrittore nel lavoro del critico; a partire da questa perentoria idea di divisione del lavoro, è disposta a discutere contenuti ideologici o episodi personali ma tende a sorvolare sulle questioni attinenti alla scrittura in sé e per sé. Eppure, è difficile non collegare la scelta di scrivere in italiano con un programmato sradicamento culturale: la metafora è forse troppo semplicistica e, sebbene quest' interpretazione emerga in alcune dichiarazioni, non ci sono sufficienti elementi per metterla alla prova. Forse, più che la sensazione del confine, nei libri di Schneider, resta quella della distanza, della frattura irriconciliabile con un mondo dell'infanzia reale che si è scoperto popolato da mostri.

Nel corso delle varie interviste da lei rilasciate in questi giorni a Barcellona, ha spesso insistito sul rapporto fra scrittura e dolore. Crede che l'atto della scrittura possa servire ad alleviare il dolore o ritiene che sia una battaglia persa sul nascere da cui il dolore ne esce peraltro rinvigorito? E poi, se scrivere di sé significa rivive- 
re certe vicende, il momento della pubblicazione non rappresenta unulteriore memoria (e quindi sofferenza) personale, seppure di secondo grado?

Sicuramente scrivere il dolore è terapeutico a patto che una persona sia in grado di farlo: significa buttar fuori il dolore. Certo, sarebbe meglio espellerlo in un diario privato, discuterne con un terapeuta per poter elaborare il lutto, dimenticare e giungere a una guarigione. Nel caso della scrittura è più difficile perché io caccio un dolore, un'infanzia frustrata e infelice per molti versi - non c'è solo la guerra: c'è anche la questione della madre - ma continuo poi a crogiuolarmici dentro perché ogni volta che vado in una scuola o in un'università in occasione della giornata della memoria logicamente ne parlo e ne risento personalmente, al punto che per altri due giorni mi resta addosso l'agitazione. Quindi, tutte le volte significa riaprire un po' perversamente una ferita. A me non fa tanto bene: ma sono legata a doppio filo ai miei libri.

Con la sua scelta di scrivere sempre in italiano, si inserisce in una scia di autori che scrivono in una lingua "altra». Viene subito da pensare a Conrad, naturalmente. Ma spostandosi sulla letteratura italiana contemporanea affiora, tra gli altri, il nome di Fleur Jaeggy. Nel caso di Helga Schneider, però, quest'alterità può anche essere letta simbolicamente - e qualche critico ha parlato di un rifiuto più che consapevole.

Io sono di madre lingua tedesca, ovviamente, ma lasciando l'Austria ho scelto di imparare benissimo l'italiano e non tanto per farne uso nella vita quotidiana: il mio obiettivo era quello di impararlo così bene da poter scrivere principalmente in italiano. Effettivamente, dopo cinque anni ho cominciato a collaborare con certi giornali ed è la mia lingua d'elezione: ormai la parlo come il tedesco o forse anche meglio. Quindi, per scelta scrivo direttamente in italiano, perché sono innamorata di questa lingua.

In Lasciami andare, madre, si nota una struttura fortemente drammatizzata che sfocia, poco dopo le prime pagine, in un dialogo stringente, quasi una figurazione verbale di una danza della morte. Nel momento della scrittura, diviene più difficile fornire la parola all'altro o il fattore memorialistico e di reportage dominano persino sull'identificazione dei personaggi?

È che, senza l'altro, il dialogo si trasforma in monologo. Non posso limitarmi a delle risposte, forse perché la mia scrittura è piuttosto coinvolgente e non mi basta la risposta dell'altro, ma devo renderne anche l'atteggiamento del viso, del corpo, e poi tutto quello che c'è intorno: i colori, i suoni, l'espressione degli occhi. Non c'è solo dialogo nel mio libro. Ci sono libri in cui c'è solo dialogo e lì bisogna immaginare la risposta dell'altro e immaginare com'è fisicamente; invece a me piace descrivere, forse in un senso letterario anche superato, con delle descrizioni anche minuziose. A me piace dare un ritmo «botta e risposta» all'interno del testo ma voglio aggiungere sempre le mie sensazioni e fornire l'impressione - anche fisica, ripeto - dell'altro. 
Nel suo dialogo con la madre cè un tentativo disperato e fallimentare di capire le ragioni della follia. Lungo i suoi scavi nel passato, qual è linterpretazione con cui oggi lei spiega il fascino che il nazismo aveva esercitato sul popolo tedesco?

È il solito vecchio culto della personalità, il culto del capo portato all'esasperazione. (Quando vedo dei vecchi film italiani, mi accorgo che anche in Italia era così. Mussolini era dappertutto: nelle poste, negli uffici pubblici, per le strade). La costruzione di un unico mito per il popolo il nazismo la cominciava dalle scuole: tutto convergeva verso il grande capo. Intorno a Hitler veniva costruito un involucro che non gli apparteneva. Ma tutti ne erano convinti; per cui, $i$ tedeschi erano sicuri che Hitler non bevesse neanche un goccio di alcol, perché era un uomo "puro». Dietro le quinte, c'era invece una persona deboluccia: un malato pieno di malattie psicosomatiche, dai problemi di stomaco a quelli di intestino. La propaganda, evidentemente, non lo diceva e non permetteva che si dicesse. E il culto del personaggio, in Germania, ha funzionato alla perfezione. In più, c'era tutto l'aspetto decorativo, come i grandi raduni organizzati durante le visite di Mussolini in Germania. Una settimana prima, gli iscritti al Partito ricevevano una circolare in cui erano invitati a partecipare alla pantomima. Era come convocare delle comparse in un film: «venite, venite a migliaia. Restate un po' lì, vi distribuiamo le bandierine e voi vi mostrate tutti sorridenti». Poi c'era la Gestapo che osservava, e qualcuno magari non sorrideva perché aveva problemi suoi, e allora la Gestapo sapeva come ricondurlo all'ordine. Era tutto teatro, ma molti ci credevano e scivolavano dentro quella follia. A parte il fatto che molti credevano veramente anche all'ideologia e sostenevano che la Germania era grande, che aveva perso la Grande Guerra per colpa degli ebrei - altrimenti, dicevano, la storia sarebbe stata incomprensibile visto che la Germania era la potenza più forte di tutto il mondo. Certo, il motivo meno plausibile per comprendere la sconfitta era proprio quello d'imputarla agli ebrei, incolpandoli di aver tramato all' ombra. Ma era questa la versione che si sentiva nelle scuole. Una sciocchezza più grande era impossibile da inventare, eppure tutti ci credevano perché avevano bisogno di un capro espiatorio.

Da un lato era stata messa in atto una revisione della storia in quella direzione; dall'altro, premeva la costruzione del mito che assumeva dei valori paganeggianti.

Certo. E tutto questo rafforzato dal terrore perché anche gli spalleggiatori a volte si stufavano. Dopo la guerra, hanno intervistato alcuni ragazzi della ex Hitlerjugend. A Norimberga, quando doveva arrivare il Führer, erano in migliaia, in uno stadio immenso. Stavano lì dalle sette a mezzogiorno, immobili, ad aspettare il Führer. Era una tortura infinita, ma loro sorridevano perché c'erano i capi della Hitlerjugend che li passavano continuamente in rivista. Cose come queste non le vedevi in un documentario, si sono sapute solo in seguito. Nel documentario, che dopo alcuni decenni è circolato anche in Italia, si vede lo stadio in giubilo, ma è solo il finale. C'era una costruzione falsissima del 
mito: il resto era condizionato dalla propaganda e da una serie di leggi che andavano persino contro gli stessi tedeschi. Quando la guerra diventò durissima, quando Goebbels gridò «Volete la guerra totale?», tutto lo Sportpalast disse di sì ma chissà quanti pensavano "no". Dopodiché, il governo prelevava i tuoi soldi dalla banca, senza dire nulla e senza che si potesse fare niente. Mia cugina era ricchissima, ma le avevano tolto tutto, per la Patria, per il Führer, contro i bolscevichi: era tutto fasullo, ma si scivolava dentro a una follia collettiva, tanto che alla fine molti ci credettero veramente.

\section{Pensa che i suoi libri possano o debbano avere una funzione pedagogica?}

Pedagogica non proprio. È vero, un giornalista italiano ha mandato una circolare a tutte le biblioteche, per dire di non trascurare il libro Stelle di cannella di Helga Schneider e ora mi chiamano in continuazione da tutte le biblioteche italiane. Ma, insomma, credo che il libro abbia un'utilità storica, è un ampliamento della storiografia ufficiale. Mi illudo di poter aggiungere un lato non solo umano, ma anche domestico e quotidiano, alla storiografia ufficiale: come spesso ho detto, è la storia raccontata dal basso. Credo che sia anche più abbordabile dai ragazzi: non racconto le grandi tappe del nazismo - Hitler dittatore, le leggi di Norimberga, i campi di concentramento, le SS, la guerra —, ma quello che succedeva veramente in quella dittatura e spero rinfreschi le idee su tutte le dittature che, in fondo, hanno sempre come base il Terrore. Se il popolo non si sente terrorizzato a sufficienza dalla dittatura, la dittatura avrà difficoltà a sussistere. Bisogna rinfrescare le idee, perché non è mica detto che non avremo mai più una dittatura. Ci può essere una dittatura che comincia subdolamente, perché la gente è poco informata. È bene che i ragazzi siano informati e che sappiano anche che non tutti i tedeschi erano necessariamente criminali, assassini o membri delle SS: c'era anche una popolazione civile che ha sofferto sotto la dittatura di Hitler, ed è gente che spesso non voleva il nazismo. Invece, sentendo parlare i ragazzi, si ha l'impressione che credano che ci fosse solo un campo di concentramento, quello di Auschwitz, - neanche Birkenau o Treblinka - ma sanno così poco e io sento il bisogno di aggiungere qualcosa storicamente alle loro informazioni. Pedagogicamente, non lo so. Forse non è un compito che spetti a me.

Nella seconda metà del secolo, in Germania si sono succeduti dei libri importanti per la consapevolezza del popolo tedesco, ma ci sono state anche parecchie situazioni di stallo in cui sembrava che agisse una sorta di autocensura collettiva.

Io compro parecchi libri tedeschi. Si scrive molto e in modo molto onesto, anche con molte testimonianze: ci sono libri storici molto limpidi. In un primissimo momento c'era stata la Trümmerliteratur, «la letteratura delle macerie», con magnifici autori che ritenevano di dover scrivere in quel momento, con l'odore dei cadaveri, perché altrimenti non avrebbero mai più potuto scrivere. Dopo, la cosa si è persa per strada: la Germania voleva rimuovere, voleva 
essere ricostruita con il piano Marshall, voleva ricominciare un nuovo capitolo - e il passato prossimo era estremamente scomodo. Solo dopo il film Holocaust c'è stato un momento in cui i giovani si sono accorti che per troppo tempo non ne avevano sentito parlare e che volevano sapere. Ed ora, in Germania, si è ripreso a parlare della tragedia con bellissimi libri, molti peraltro scritti da ebrei. 